\title{
É possível prever o número de nebulizações e o uso de corticosteróide intravenoso em crianças com crise asmática na unidade de emergência?
}

\author{
Is it possible to predict the number of nebulizations and the use of intravenous corticosteroid \\ in children with asthmatic attack in the emergency room?
}

\author{
Maria L.Z. Paro ${ }^{1}$, Joaquim C. Rodrigues ${ }^{2}$
}

\begin{abstract}
Resumo
Objetivo: identificar o número de nebulizações necessárias e a demanda de corticosteróide intravenoso em crianças em crise asmática, a partir de características clínicas e funcionais observadas no momento da admissão na unidade de emergência.

Métodos: foram avaliadas prospectivamente 130 crianças em crise asmática, na faixa etária de 1 a 13 anos. No momento da admissão, as crianças foram avaliadas por meio de escore clínico e medidas de saturação arterial de oxigênio (por oximetria de pulso) e de pico de fluxo expiratório. Em seguida, receberam um tratamento padronizado e foram acompanhadas em relação à necessidade de nebulizações consecutivas com $\beta 2$ agonista e costicosteróide intravenoso. Através de análise de regressão buscou-se uma correlação dos parâmetros avaliados com o número de nebulizações realizadas e o uso de corticosteróide.

Resultados: oitenta e oito crianças $(67,7 \%)$ receberam de uma até três nebulizações, e 42 crianças $(32,3 \%)$ receberam seis nebulizações. Sessenta e oito crianças $(52,3 \%)$ receberam corticosteróide. Os valores iniciais de escore clínico, saturação arterial de oxigênio e pico de fluxo expiratório mostraram uma correlação significativa com o número de nebulizações realizadas e com a necessidade do uso de corticosteróide.

Conclusão: com base em nossos resultados, é possível predizer e antecipar, no momento da admissão das crianças em crise asmática na unidade de emergência, a necessidade do uso de corticosteróide e de um maior número de nebulizações, o que pode alterar o prognóstico e o tempo de evolução da crise.
\end{abstract}

J Pediatr (Rio J) 2001; 77 (3): 219-26: asma, criança, asma aguda, tratamento, corticosteróide, 32 agonista.

\section{Introdução}

A asma é uma importante causa de atendimento pediátrico nos serviços de emergência, sendo responsável por

1. Mestre em Pediatria - FMUSP.

2. Mestre e Doutor em Pediatria - FMUSP. Médico Chefe da Unidade de Pneumologia do Instituto da Criança do Hospital das Clínicas - FMUSP. Fonte financiadora: FAPESP.

\begin{abstract}
Objective: to identify the number of nebulizations needed and the demand for intravenous corticosteroids in children with asthmatic attack, considering clinical and functional characteristics presented at the moment children were admitted to the emergency room.
\end{abstract}

Methods: we prospectively evaluated 130 children with asthmatic attack and from 1 to 13 years of age. At the moment children were admitted, they were evaluated according to clinical score, arterial oxygen saturation (pulse oximetry), and peak expiratory flow. Next, children received a standard treatment and were observed for the number of consecutive nebulizations required and the use of corticosteroid. Using regression analysis, we assessed the parameters evaluated for a correlation with the number of nebulizations and the use of intravenous corticosteroid.

Results: eighty-eight children $(67.7 \%)$ received from 1 to 3 nebulizations and 42 children (32.3\%), 6 nebulizations. Sixty-eight children $(52.3 \%)$ received corticosteroid. The initial values for clinical scores, arterial oxygen saturation, and peak expiratory flow showed a significant correlation with the number of nebulizations required and the need for corticosteroids.

Conclusions: according to our results, it is possible to predict and anticipate, at the time chidren are admitted with asthmatic attack, the need for corticosteroid and more nebulizations, which can change the prognosis and the time of evolution of the attack.

J Pediatr (Rio J) 2001; 77 (3): 219-26: asthma, child, acute asthma, treatment, corticosteroid, $\beta 2$ agonist.

uma parcela significativa das hospitalizações ${ }^{1,2}$. Constitui um sério problema de saúde pública em todo o mundo e tem sido motivo de muita discussão no meio científico, pois, apesar dos recentes avanços no conhecimento de sua patogênese, a prevalência, a morbidade e a mortalidade têm aumentado progressivamente nos últimos vinte anos ${ }^{1-3}$. 
Cerca de 70\% das mortes por asma ocorrem durante a hospitalização, o que vem produzindo um importante consenso de que a maioria dos pacientes morre porque a gravidade da doença é subestimada e, conseqüentemente, a doença é tratada de forma insuficiente ou inadequada ${ }^{1,4,5}$.

$\mathrm{O}$ tratamento da asma nos serviços de atendimento de emergência sempre envolve decisões potencialmente difíceis como dispensar o paciente, continuar o tratamento ou hospitalizar. Na maioria das vezes, essa decisão é feita de forma subjetiva, baseada na experiência clínica do médico que assiste à criança. Nos últimos anos, a oximetria de pulso e a medida do pico de fluxo expiratório têm sido recomendadas na monitorização da gravidade da crise asmática e da resposta ao tratamento, na tentativa de aprimorar o atendimento da crise asmática ${ }^{6-14}$.

Os Consensos no Manejo da Asma ${ }^{15-17}$ têm recomendado tratamento agressivo na crise asmática, destacando a necessidade de doses elevadas de broncodilatadores e a utilização precoce de corticosteróide. Entretanto, o uso insuficiente de corticosteróides e de subdoses ou grandes intervalos entre as nebulizações com $\beta 2$ agonistas estão entre os problemas apontados no manejo da asma no Pronto-Socorro ${ }^{15}$. Nós planejamos este estudo com o objetivo de verificar se anormalidades clínicas e funcionais, observadas no momento da admissão de crianças asmáticas na Unidade de Emergência (UE), poderiam ser preditivas do número de nebulizações que seriam necessárias para reverter a obstrução brônquica, bem como da necessidade de utilização de corticosteróide endovenoso.

\section{Métodos}

Foram estudadas prospectivamente 130 crianças em crise asmática, na faixa etária de 1 a 13 anos, admitidas na Unidade de Emergência Pediátrica do Hospital de Base de
São José do Rio Preto, no período de setembro de 1996 a maio de 1997.

Como critério de inclusão no estudo, considerou-se a recomendação da American Thoracic Society ${ }^{18}$, ou seja, a criança foi considerada asmática quando apresentava história familiar de atopia e pelo menos dois episódios anteriores de crise de sibilância, com melhora clínica após uso de broncodilatador oral, parenteral ou inalatório. A crise no momento da admissão foi caracterizada pela presença auscultatória de sibilos predominantemente expiratórios. Foram excluídas as crianças portadoras de hemoglobinopatias, bronquiolite aguda, cardiopatias congênitas ou adquiridas, fibrose cística, displasia broncopulmonar e as crianças menores de um ano de idade. $\mathrm{O}$ projeto de pesquisa foi avaliado e aprovado pela Comissão de Ética Médica da Faculdade de Medicina de São José do Rio Preto e foi obtido consentimento dos pais ou responsáveis pela criança para a inclusão da mesma no protocolo.

Inicialmente, antes de qualquer tratamento, o autor realizou o exame físico e a medida da saturação arterial de oxigênio $\left(\mathrm{SaO}_{2}\right)$ em todas as crianças e a medida do pico de fluxo expiratório (PFE) nas crianças acima de cinco anos de idade. Da avaliação clínica, foi obtido um escore clínico modificado a partir do escore de Wood ${ }^{19}$, que incluiu freqüência respiratória, cor, uso de musculatura acessória, ausculta pulmonar e função cerebral. Cada um desses itens foi pontuado de 0 a 2 , conforme a intensidade do sintoma, e a soma de todos gerou o escore do paciente no momento da avaliação. A Tabela 1 mostra o escore clínico utilizado.

$\mathrm{A} \mathrm{SaO}_{2}$ foi mensurada por um oxímetro de pulso (Dixtal Biomédica Ltd., DX 2405, Manaus, AMl) conectado ao dedo indicador ou halux da criança, por um sensor de tamanho apropriado para a idade, em ar ambiente. $\mathrm{A} \mathrm{SaO}_{2}$ foi registrada após o primeiro minuto de estabilização, como o valor que permaneceu mais constante durante o

Tabela 1 - Escore clínico para avaliação da gravidade da crise asmática em crianças na Unidade de Emergência

\begin{tabular}{lccc}
\hline & $\mathbf{0}$ & $\mathbf{1}$ & $\mathbf{2}$ \\
\hline Cor & normal & palidez & cicianose \\
Freqüência respiratória (ipm)* & & & \\
de 1 a 2 anos & até 30 & $31-60$ & acima de 60 \\
de 2 a 5 anos & até 25 & $26-50$ & acima de 50 \\
acima de 5 anos & até 20 & $21-40$ & acima de 40 \\
Uso de musculatura acessória & nenhum & leve ou moderado & intenso \\
Ausculta pulmonar & sibilos no final & sibilos ins e & ausência \\
da inspiração & expiratórios & de sons \\
Função cerebral & normal & deprimido & comatoso \\
& & ou agitado & \\
\hline
\end{tabular}

* Os valores normais foram obtidos de Waring ${ }^{28}$ 
segundo minuto. $\mathrm{O}$ valor do PFE foi obtido por meio de um aparelho portátil, o Peak Flow Meter de Wright (Clement Clarke International Ltda., London, England). Para a medida do PFE, o autor solicitou que a criança ficasse em posição ortostática, sem dobrar a região cervical, e segurasse o aparelho horizontalmente, cuidando para manter as saídas de ar desobstruídas ${ }^{20}$. A criança então realizava uma inspiração profunda, colocava o aparelho na boca e fechava os lábios em torno do bocal. Em seguida, expirava forte e rapidamente. Essa manobra foi repetida por três vezes, sendo registrado o maior valor obtido nas três manobras, expresso em valor absoluto ${ }^{20,21}$. Posteriormente, esse valor foi expresso em porcentagem do valor previsto para a altura e sexo do paciente, utilizando-se a equação de Torres et al. ${ }^{22}$

Após a avaliação inicial, a terapêutica e os controles foram instituídos, seguindo as recomendações internacionais estabelecidas em $1995^{2}$. Os pacientes receberam nebulizações intermitentes com $0,15 \mathrm{mg} / \mathrm{kg}$ de uma solução de salbutamol a $0,5 \%$, adicionado a $5 \mathrm{ml}$ de solução salina a $0,9 \%$, com fluxo de oxigênio de seis litros por minuto. Os pacientes admitidos com $\mathrm{SaO}_{2}$ abaixo de $91 \%$, ou em crise grave, ou com redução do nível de consciência, receberam previamente $0,01 \mathrm{ml} / \mathrm{kg}$ de terbutalina por via subcutânea. De acordo com a resposta clínica do paciente e da $\mathrm{SaO}_{2}$, as nebulizações foram repetidas a cada vinte minutos, até no máximo duas horas após o início do tratamento, tal como preconizado pelas normas internacionais de $1995^{2}$. Como critério objetivo, o paciente deveria apresentar níveis de $\mathrm{SaO}_{2} \geq 95 \%$ para que a resposta à nebulização fosse considerada satisfatória. O corticosteróide intravenoso foi indicado em dois momentos: após a primeira nebulização, quando o paciente não apresentava nenhuma melhora clínica, ou após a terceira nebulização, caso apresentasse melhora clínica parcial, $\mathrm{com} \mathrm{SaO}_{2}<95 \%$. Em uma dessas duas situações foi administrada uma dose endovenosa de metilprednisolona de $2 \mathrm{mg} / \mathrm{kg}$.

Os pacientes foram avaliados cinco minutos após a primeira, terceira e sexta nebulizações, durante o tempo de permanência do paciente no protocolo de estudo. A cada momento de avaliação, o autor registrou os valores do escore clínico, da $\mathrm{SaO}_{2}$ em ar ambiente e do PFE. Considerou-se a alta para o domicílio, independentemente do número de nebulizações, após melhora clínica marcada e $\mathrm{SaO}_{2} \geq$ $95 \%$. Indicou-se hospitalização quando, após um número máximo de 6 nebulizações consecutivas, não houve melhora clínica satisfatória, sendo mantida a $\mathrm{SaO}_{2}$ abaixo de $95 \%$. Nos pacientes com $\mathrm{SaO}_{2} \leq 91 \%$ após a terceira nebulização, a indicação de hospitalização foi mais precoce.

A análise estatística incluiu a técnica de análise de variância, para avaliar o comportamento médio das variáveis aferidas no momento da admissão na UE, de acordo com o número de nebulizações recebidas pelos pacientes e de acordo com o uso de corticosteróide na Emergência. Quando a diferença entre as médias dos parâmetros estudados foi significante entre os grupos que receberam uma, três ou seis nebulizações, prosseguiu-se a análise com as comparações múltiplas de Tukey ${ }^{23}$. Por meio de análise de regressão, foi possível criar equações correlacionando as variáveis estudadas com o número de nebulizações e com o uso de corticosteróide. Posteriormente, calculamos a sensibilidade, a especificidade e os valores preditivos positivo e negativo, usando diferentes níveis iniciais de escore clínico, $\mathrm{SaO}_{2} \mathrm{e}$ PFE, para predizer a necessidade do uso de corticosteróide na UE.

\section{Resultados}

Nesta casuística, a proporção entre as crianças do sexo masculino e feminino foi de $1,8: 1$. As médias e os desviospadrão para idade (meses), altura $(\mathrm{cm})$ e peso $(\mathrm{kg})$ foram, respectivamente, $68,67 \pm 39,0,111,09 \pm 20,34$ e 21,24 \pm 9,18. O PFE foi obtido somente em 56 crianças, acima de 5 anos (87,5\%), sendo que 8 crianças nesta faixa etária não efetuaram a manobra, por falta de colaboração ou por incapacidade pela gravidade da crise.

Oitenta e quatro crianças $(64,6 \%)$ já haviam recebido tratamento domiciliar com broncodilatador oral ou inalatório, antes de serem admitidas no estudo. Dez crianças receberam terbutalina subcutânea no momento da admissão na UE, mas somente após a avaliação inicial.

Trinta e oito crianças $(29,2 \%)$ receberam uma nebulização com 32 agonista, 50 crianças $(38,5 \%)$ receberam 3 nebulizações e 42 crianças $(32,3 \%)$ receberam 6 nebulizações. Dentre as que necessitaram de 6 nebulizações, aparentemente as de maior gravidade, 13 foram internadas e 29 tiveram alta da UE.

A Tabela 2 mostra as médias e os intervalos de confiança de escore clínico, de $\mathrm{SaO}_{2}$ e de \% PFE iniciais (valores obtidos no momento da admissão da criança na UE), de acordo com o número de nebulizações efetuadas durante o período de permanência das crianças na UE. A média de escore clínico inicial, entre os pacientes que fizeram uma nebulização, foi mais baixa do que a dos que fizeram três ou seis nebulizações. Na análise estatística pelo teste de Tukey, houve diferença significante entre os grupos que fizeram 1 , 3 e 6 nebulizações $(\mathrm{p}<0,05)$. A média de $\mathrm{SaO}_{2}$ inicial entre os pacientes que fizeram uma nebulização foi mais alta do que a dos que fizeram três ou seis nebulizações. $\mathrm{Na}$ análise estatística pelo teste de Tukey, observamos diferenças estatisticamente significantes entre os que fizeram 1 ou 3 nebulizações, quando comparados aos que fizeram 6 nebulizações $(p<0,05)$. Não houve diferença significante entre as médias de $\mathrm{SaO}_{2}$ dos que fizeram 1 e 3 nebulizações ( $p>0,05)$. A média de \% PFE inicial entre os pacientes que fizeram uma nebulização com $\beta 2$ agonista foi mais alta do que a dos que fizeram três ou seis nebulizações. Na análise estatística destes resultados pelo teste de Tukey, observamos diferenças estatisticamente significantes entre os que fizeram 1 ou 3 nebulizações quando comparados aos que fizeram 6 nebulizações $(p<0,05)$. Não houve diferença 
Tabela 2 - Distribuição das médias e intervalos de confiança de escore clínico, saturação arterial de oxigênio $\left(\mathrm{SaO}_{2}\right)$ e pico de fluxo expiratório (PFE) iniciais, de acordo com o número de nebulizações com salbutamol, realizadas durante o período de permanência na Emergência

\begin{tabular}{|c|c|c|c|c|c|}
\hline & \multicolumn{5}{|c|}{ Número de nebulizações } \\
\hline & 1 & & 3 & & 6 \\
\hline $\begin{array}{l}\text { Escore clínico } \\
(\mathrm{n}=130)\end{array}$ & $2,1(1,86-2,34)$ & $\dagger$ & $3,3(2,9-3,7)$ & $\dagger$ & $4,6(4,08-5,12)$ \\
\hline $\begin{array}{c}\mathbf{S a O}_{2}(\%) \\
(\mathrm{n}=130)\end{array}$ & $96,1(95,76-96,44)$ & $*$ & $95,2(94,74-95,66)$ & $\dagger$ & $93,6(92,74-94,46)$ \\
\hline $\begin{array}{c}\text { \% PFE } \\
(\mathrm{n}=56)\end{array}$ & $68,6(59,91-77,29)$ & $*$ & $59,7(49,12-70,28)$ & $\dagger$ & $37,4(29,46-45,34)$ \\
\hline
\end{tabular}

\footnotetext{
( ) = média (intervalo de confiança de 95\% para a média)

$\dagger=\mathrm{p}<0,05$ (diferença estatisticamente significante)

* = $p>0,05$ (diferença estatisticamente não significante)

nota: na análise estatística foi aplicado o teste de comparações múltiplas de Tukey
}

significante entre os grupos que fizeram 1 e 3 nebulizações $(\mathrm{p}>0,05)$.

Através da análise de regressão, foi possível criar equações para estimar-se o número total de nebulizações necessárias, de acordo com os valores de escore clínico, de $\mathrm{SaO}_{2}$ e de \% PFE, obtidos no momento da admissão na UE. Todas as três variáveis mostraram correlação significativa com o número de nebulizações administradas na UE ( $\mathrm{p}<0,0001)$. Também foi criada uma equação para estimar-se o número total de nebulizações necessárias, de acordo com os valores de escore clínico e $\mathrm{SaO}_{2}$ combinados. As equações obtidas por regressão logística, onde $\mathrm{N}$ é o número de nebulizações previstas, com seus respectivos coeficientes de correlação $\mathrm{R}^{2}$, são as seguintes: 1) $\mathrm{N}=1,09+0,679$ x escore clínico $\left.\left.\left(\mathrm{R}^{2}=0,329\right) ; 2\right) \mathrm{N}=41,8-0,404 \times \mathrm{SaO}_{2}\left(\mathrm{R}^{2}=0,207\right) ; 3\right)$ $\mathrm{N}=5,26-0,0420 \times \%$ PFE previsto $\left.\left(\mathrm{R}^{2}=0,260\right) ; 4\right) \mathrm{N}=23,5$ $+0,551 \times$ escore $-0,232 \times \mathrm{SaO}_{2}\left(\mathrm{R}^{2}=0,386\right)$.

Através da aplicação dessas equações, foi possível estabelecer uma regra prática que permite estimar o número aproximado de nebulizações com $\beta 2$ agonista, baseado nos valores iniciais de escore clínico, $\mathrm{SaO}_{2} \mathrm{e} \%$ PFE. Essa regra prática é apresentada na Tabela 3.

Sessenta e oito crianças $(52,3 \%)$ receberam corticosteróide (metilprednisolona endovenosa), durante o tratamento na UE. A Tabela 4 mostra as médias e intervalos de confiança de escore clínico, $\mathrm{SaO}_{2}$ e \% PFE iniciais das crianças, de acordo com a necessidade do uso de corticosteróide, durante o período de tratamento da crise de asma na UE. A média de escore clínico inicial, entre os pacientes que receberam corticosteróide, foi mais alta do que a dos pacientes que não receberam. $\mathrm{Na}$ análise de variância, a diferença entre as médias foi altamente significante $(\mathrm{p}<0,001)$. A média de $\mathrm{SaO}_{2}$ inicial, entre os pacientes que receberam corticosteróide na Emergência, foi mais baixa do que a dos pacientes que não receberam. Na análise de variância, a diferença entre as médias foi altamente significante $(p<0,001)$. Os resultados mostraram que a média de \% PFE inicial, entre os pacientes que receberam corticosteróide na UE, foi mais baixa do que a dos que não receberam. A análise de variância mostrou que a diferença entre as médias foi significante $(\mathrm{p}=0,004)$.

Através de análise de regressão foi possível encontrar funções para predizer o uso de corticosteróide na UE, baseando-se nos valores de escore clínico, de $\mathrm{SaO}_{2}$ e de \%

Tabela 3 - Estimativa do número de nebulizações na Unidade de Emergência, baseada nos valores iniciais de escore clínico, $\mathrm{SaO}_{2}$ e \% do PFE na casuística estudada

\begin{tabular}{lccc}
\hline $\mathrm{SaO}_{\mathbf{2}}(\%)$ & escore & $\%$ PFE & $\begin{array}{c}\text { númerode } \\
\text { nebulizações }\end{array}$ \\
\hline$\geq 97$ & $0|-| 2$ & $\geq 78$ & 1 \\
$94 \mid-97$ & $2-\mid 4$ & $50 \mid-78$ & 3 \\
$\leq 93$ & $\geq 5$ & $<50$ & 6 \\
\hline
\end{tabular}

$\mathrm{SaO}_{2}=$ saturação arterial de oxigênio

$\%$ PFE = pico de fluxo expiratório 
Tabela 4 - Distribuição das médias e intervalos de confiança de escore clínico, saturação arterial de oxigênio $\left(\mathrm{SaO}_{2}\right)$ e pico de fluxo expiratório (PFE) iniciais, de acordo com a necessidade do uso de corticosteróide durante o tratamento na Emergência

\begin{tabular}{lccc}
\hline & \multicolumn{2}{c}{ Uso de corticosteróide } & p * \\
\hline Parâmetros & Sim & Não & \\
\hline SaO $_{2}(\%)(\mathrm{n}=130)$ & $94,1(93,48-94,72)$ & $95,9(95,60-96,20)$ & $<0,001$ \\
Escore clínico $(\mathrm{n}=130)$ & $4,1(3,71-4,49)$ & $2,6(2,27-2,93)$ & $<0,001$ \\
\% PFE $(\mathrm{n}=56)$ & $46,28(37,12-55,44)$ & $65,27(57,71-72,83)$ & 0,004 \\
\hline
\end{tabular}

( ) = média (intervalo de confiança de 95\% para a média)

* $p=$ valor de significância na análise de variância

PFE obtidos no momento da admissão e, ainda, na associação desses parâmetros laboratoriais. Estas funções, com suas respectivas taxas de acerto (TA) e taxa de erro (TE), estão representadas na Tabela 5. Observou-se que as taxas de erro e de acerto para as funções obtidas para a predição do uso de corticosteróide, baseadas em escore e $\mathrm{SaO}_{2}$ foram bastante semelhantes. Para o PFE, a taxa de acerto foi um pouco mais alta, mas também houve aumento da taxa de erro. Através da aplicação dessas equações, foi possível estabelecer uma regra prática que permite predizer a necessidade do uso de corticosteróide com base nos valores da $\mathrm{SaO}_{2}$, do escore clínico e do \% PFE, obtidos no momento da admissão na UE. Essa regra prática é descrita na Tabela 6.

Tabela 5 - Representação das funções obtidas por análise de regressão e suas respectivas taxas de acerto e de erro, para predizer a necessidade de corticosteróide, baseando-se nos valores iniciais de escore clínico, $\mathrm{SaO}_{2}$ e \% PFE

\begin{tabular}{lcc}
\hline Função* & TA(\%) & TE(\%) \\
\hline 0,69 x escore clínico - 2,15 & 67,2 & 15,3 \\
47,83 - 0,50 x SaO & 65,1 & 19,8 \\
1,62 - 0,04 x \%PFE & 74,2 & 25,3 \\
39,77 + 0,60 x escore - 0,44 x SaO & \\
99,38 + 0,83 x escore - 1,08 x $\mathrm{SaO}_{2}+$ & 80,1 & 17,0 \\
0,004 x \%PFE & 90,8 & \\
\end{tabular}

* Nota: sempre que o resultado da função for maior do que zero, existe indicação para o uso de corticosteróide na UE

$\mathrm{SaO}_{2}=$ saturação arterial de oxigênio

$\%$ PFE $=$ pico de fluxo expiratório
Utilizando vários níveis de corte para os valores de escore clínico, $\mathrm{SaO}_{2}$ e \% PFE no momento da admissão na $\mathrm{UE}$, foram calculados a sensibilidade, a especificidade, os valores preditivos positivos (VPP) e os valores preditivos negativos (VPN) para estimar a necessidade do uso de corticosteróide. Esses resultados são apresentados na Tabela 7 .

Tabela 6 - Previsão da utilização de corticosteróide na Unidade de Emergência, baseada nos valores iniciais de escore clínico, $\mathrm{SaO}_{2}$ e \% do PFE na casuística estudada

\begin{tabular}{cccc}
\hline $\mathrm{SaO}_{\mathbf{2}}(\%)$ & escore & \% PFE & uso de corticosteróide \\
\hline$<95$ & $\geq 4$ & $\leq 40$ & sim \\
$\geq 95$ & $<4$ & $>40$ & não \\
\hline
\end{tabular}

$\mathrm{SaO}_{2}=$ saturação arterial de oxigênio

$\%$ PFE = pico de fluxo expiratório

\section{Discussão}

Mais de um quarto dos pacientes com crise de asma que são dispensados para o domicílio, após atendimento em $\mathrm{UE}$, apresentam recidiva do quadro e retornam à $\mathrm{UE}^{17}$. Esse dado provavelmente reflete a falta de um tratamento padronizado e adequado da crise asmática.

A abordagem da crise asmática, segundo as normas preconizadas internacionalmente ${ }^{2}$, obedece a um organograma de nebulizações consecutivas com $\beta 2$ agonista, a cada 20 minutos, até um máximo de seis, o que envolve a necessidade de reavaliações freqüentes. Em uma UE, as decisões devem ser definidas de forma rápida e eficiente ao mesmo tempo. Muitas vezes, em função da demanda de atendimento, as condutas terapêuticas são retardadas. Do 
Tabela 7 - Distribuição da sensibilidade, da especificidade e dos valores preditivos positivo e negativo, usando diferentes níveis iniciais de escore clínico, saturação arterial de oxigênio $\left(\mathrm{SaO}_{2}\right)$ e pico de fluxo expiratório (PFE), para predizer o uso de corticosteróide no tratamento da crise de asma

\begin{tabular}{rccccc}
\hline & $\mathbf{N}^{\circ}$ de pacientes & $\mathbf{S}(\boldsymbol{\%})$ & $\mathbf{E}(\boldsymbol{\%})$ & VPP $(\boldsymbol{\%})$ & VPN $(\boldsymbol{\%})$ \\
\hline Escore & & & & & \\
$\geq 7$ & 08 & 11,8 & 100,0 & 100,0 & 50,8 \\
$\geq 6$ & 18 & 22,1 & 95,2 & 83,3 & 52,7 \\
$\geq 4$ & 45 & 50,0 & 82,2 & 75,6 & 60,0 \\
$\geq 2$ & 117 & 98,5 & 19,4 & 57,3 & 92,3 \\
& & & & & \\
$\mathbf{S a O}_{\mathbf{2}}(\boldsymbol{\%})$ & & & & & \\
$<91$ & 08 & 11,8 & 100,0 & 100,0 & 50,8 \\
$<93$ & 19 & 26,5 & 98,4 & 94,7 & 54,9 \\
$<95$ & 41 & 50,0 & 89,7 & 82,9 & 61,8 \\
$<97$ & 99 & 83,8 & 32,3 & 57,6 & 64,5 \\
$\mathbf{\% ~ P F E ~}$ & & & & & \\
$\leq 30$ & 07 & 20,0 & 91,7 & 57,1 & 67,3 \\
$\leq 40$ & 14 & 40,0 & 83,3 & 57,1 & 71,4 \\
$\leq 60$ & 29 & 75,0 & 61,1 & 51,7 & 81,5 \\
$\leq 80$ & 46 & 95,0 & 25,0 & 41,3 & 90,0 \\
\hline
\end{tabular}

$\mathrm{S}=$ sensibilidade VPP $=$ valor preditivo positivo

$\mathrm{E}=$ especificidade $\mathrm{VPN}=$ valor preditivo negativo

ponto de vista prático, é interessante tentar definir o tratamento de uma criança em crise asmática, em relação ao número de nebulizações com $\$ 2$ agonista e ao uso de corticosteróide endovenoso, no momento da admissão da mesma na UE.

Em nosso estudo, observamos uma correlação entre o número de nebulizações necessárias para o alívio da crise e o aumento dos valores iniciais de escore clínico e a queda dos valores iniciais de $\mathrm{SaO}_{2}$ e PFE. Observações semelhantes foram feitas por Yamamoto et al. ${ }^{24}$, que avaliaram 7.381 pacientes menores de 21 anos, em crise de asma. Eles verificaram que as médias de $\mathrm{SaO}_{2}$ iniciais foram progressivamente menores nos pacientes que necessitaram de maior número de tratamentos com 32 agonista na UE. Os mesmos autores fizeram observações semelhantes em um outro estudo, que envolveu 785 asmáticos, na mesma faixa etária $^{25}$, no qual também se avaliou o PFE. Observaram que as médias de PFE iniciais também foram progressivamente menores nos pacientes que necessitaram de maior número de tratamentos com broncodilatador na UE.

Em nosso estudo, foi possível obter equações de regressão, correlacionando o número de nebulizações com os parâmetros estudados (escore clínico, $\mathrm{SaO}_{2}$ e PFE). No entanto, os coeficientes de determinação $\left(\mathrm{R}^{2}\right)$ dessas equações foram baixos, denotando pouco poder explicativo da variação total encontrada. Houve um coeficiente mais alto quando a equação foi obtida a partir da associação de escore e saturação, mas ainda assim, o valor encontrado foi relativamente baixo $(38,6 \%)$. Apesar disso, sugerimos que crianças admitidas com escore maior ou igual a $3, \mathrm{SaO}_{2}$ menor ou igual a $96 \%$ e PFE abaixo de $50 \%$ do previsto, sejam candidatas à administração de pelo menos 3 nebulizações intermitentes com ß-2 agonista.

No estudo de Yamamoto et al. ${ }^{24}$, realizou-se uma regressão linear para medir o grau de correlação entre o número total de tratamentos efetuados com broncodilatador e os valores iniciais de $\mathrm{SaO}_{2}$ e PFE. As duas variáveis mostraram correlação significativa com o número de nebulizações $(\mathrm{p}<0,0001)$, com valores de $\mathrm{R}^{2}$ também baixos (18,3\% e $14,3 \%$, respectivamente).

Em nosso estudo, utilizando-se de critérios preconizados internacionalmente ${ }^{2}, 52,3 \%$ dos pacientes receberam corticosteróide endovenoso durante o atendimento na UE. Observamos que a média de escore clínico inicial dos pacientes que receberam corticosteróide foi significativamente maior do que a média dos pacientes que não receberam, e que as médias de $\mathrm{SaO}_{2}$ e de PFE iniciais foram significativamente menores nos pacientes que necessitaram de corticosteróide. Verificamos que $100 \%$ das crianças que foram admitidas na UE com um escore clínico inicial igual ou acima de 7 e uma $\mathrm{SaO}_{2}$ abaixo de $91 \%$ receberam corticosteróide. Não encontramos na literatura nenhum 
trabalho sobre os critérios para indicação de corticosteróide no tratamento da crise asmática, baseando-se nos valores iniciais de escore clínico, $\mathrm{SaO}_{2}$ e PFE.

A partir dos resultados do nosso estudo, é possível sugerir que o uso de corticosteróide na crise asmática seja considerado para toda criança com valores iniciais de escore clínico igual ou acima de 4, de $\mathrm{SaO}_{2}$ abaixo de $95 \%$ e de PFE igual ou abaixo de $40 \%$ do previsto. Sugere-se, pela análise estatística, que a presença de 2 ou 3 destes critérios reforça a necessidade do uso precoce de corticosteróide. Esses níveis foram obtidos pela análise de regressão e apresentaram altos valores preditivos positivos (VPP) e de especificidade, para indicar a necessidade de corticosteróide. Com este critério é possível antecipar a introdução de corticosteróide no momento da admissão da criança asmática na UE, o que pode contribuir para uma melhora precoce na função pulmonar do paciente ${ }^{26}$.

Estudos de metanálise avaliando os benefícios do tratamento com corticosteróides em UE demonstram diminuição significativa nas taxas de internação e no número de recidivas após cinco a sete dias do atendimento da crise asmática ${ }^{27}$. Considerando que o corticosteróide tem início de ação demorado, a antecipação do seu uso pode ser extremamente benéfica na evolução da crise asmática. $\mathrm{O}$ retardo na indicação de utilização dos corticosteróides pode alterar o prognóstico da crise asmática em crianças, sendo estes de importância fundamental na resolução da reagudização e intensificação do processo inflamatório ${ }^{15}$.

O exame físico da criança é de grande importância na avaliação da crise asmática. Entretanto, pode não ser suficiente, e muitas vezes o pediatra se questiona quanto a usar corticosteróide endovenoso ou não e quanto a nebulizar uma ou mais vezes. Por meio de medidas simples e não invasivas, como a oximetria de pulso e o pico de fluxo expiratório, podemos melhorar a avaliação da intensidade de uma crise asmática e, ainda, definir a terapêutica. Talvez dessa forma o atendimento da crise asmática possa ser otimizado.

Com base em nosso estudo, recomendamos que as medidas de saturação arterial de oxigênio e de pico de fluxo expiratório, obtidas no momento da admissão das crianças em crise asmática na UE, sejam aplicadas às decisões terapêuticas, principalmente em relação à utilização de corticosteróide, o que pode alterar o prognóstico e o tempo de evolução da crise.

\section{Agradecimentos}

À Fundação de Amparo à Pesquisa do Estado de São Paulo (FAPESP), por ter sido a fonte financiadora da pesquisa.

\section{Referências bibliográficas}

1. Buist AS, Vollmer WM. Reflections on the rise in asthma morbidity and mortality. JAMA 1990;264:1719-20.

2. Sheffer AL, Bartal M, Bousquet J. Global initiative for asthma: global strategy for asthma management and prevention. NHLBI/ WHO Workshop Report. Bethesda: National Institutes of Health; 1995.

3. Jonasson G, Lodrup Carlsen KC, Leegaard J, Carlsen KH, Mowinckel P, Halvorsen KS. Trends in hospital admissions for childhood asthma in Oslo, Norway, 1980-95. Allergy 2000;55:232-9.

4. Saetta M, Stefano AD, Rosina C, Thiene G, Fabbri LM. Quantitative structural analysis of peripheral airways and arteries in sudden fatal asthma. Am Rev Respir Dis 1991;143:138-43.

5. Evans R, Mullaly DI, Wilson RW. National trends in the morbidity and mortality of asthma in the U.S. Prevalence, hospitalization and death from asthma over two decades: 1965-1984. Chest 1987;91:65-74.

6. Baker MD. Pitfalls in the use of clinical asthma scoring. Am J Dis Child 1988;142:183-5.

7. Sole D, Komatsu MK, Carvalho KV, Naspitz CK. Pulse oximetry in the evaluation of the severety of acute asthma and/or wheezing in children. J Asthma 1999;36:327-33.

8. Bishop J, Nolan T. Pulse oximetry in acute asthma. Arch Dis Child 1991;66:724-5.

9. Bolliger CT, Fourie PR, Kotze D, Joubert JR. Relation of measures of asthma and response to treatment to outcome in acute severe asthma. Thorax 1992;47:943-7.

10. Connett GJ, Lenney W. Use of pulse oximetry in the hospital management of acute asthma in childhood. Pediatr Pulmonol 1993;15:345-9.

11. Geelhoed GC, Landau LI, LeSouef PN. Oximetry and peak expiratory flow in assessment of acute childhood asthma. J Pediatr 1990;117:907-9.

12. Kerem E, Tibshirani R, Canny G, Bentur L, Reisman J, Schuh S, et al. Predicting the need for hospitalization in children with acute asthma. Chest 1990;98:1355-61.

13. Mayefsky JH, El-Shinaway Y. The usefulness of pulse oximetry in evaluating acutely ill asthmatics. Pediatr Emerg Care 1992;8:262-4.

14. Nowak RM, Tomlanovich MC, Sarkar, DD, Kuale PA, Anderson JA. Arterial blood gases and pulmonary function testing in acute bronchial asthma: predicting patient outcomes. JAMA 1983;249:2043-6.

15. II Consenso Brasileiro no Manejo da Asma. J Pneumol 1998;24:171-276.

16. National Heart, Lung and Blood Institute. International consensus report on diagnosis and treatment of asthma. Washington, DC: Department of Health and Human Services; 1992.

17. National Heart, Lung and Blood Institute. Guidelines for the diagnosis and management of asthma. In: National Asthma Education Program. Expert Panel Report II. Bethesda, 1997.

18. American Thoracic Society. Definitions and classifications of chronic bronchitis, asthma and pulmonary emphysema. Am Rev Respir Dis 1962;85:762-4.

19. Wood DW, Downes JJ, Lecks HJ. A clinical scoring system for diagnosis of respiratory failure. Am J Dis Child 1972;123:227-8.

20. Perks WH, Tams JP, Thompson DA, Prowse K. An evaluation of the mini-Wright peak flow meter. Thorax 1979;34:79-81.

21. Quakenboss JJ, Lebowitz MD, Krzyzanowski M. The normal range of diurnal changes in peak expiratory flow rates: Relationship to symptoms and respiratory disease. Am Rev Respir Dis 1990;143:323-30. 
22. Torres LAGMM, Pereira GA, Piovesan MI, Telles MJV, Rahal REB, Manço JC. Valores de referência para o fluxo expiratório máximo em crianças de 5 a 12 anos de idade. J Pneumol 1997;23(Supl):3. [Apresentado no VII Congresso Brasileiro de Pneumologia Pediátrica, Blumenau, 1997]

23. Rosner B. Fundamentals of biostatistics. 2nd ed. Boston: Duxbury Press; 1986.

24. Yamamoto LG, Wiebe RA, Anaya C, Chang RKS, Chang MA, Terada AM, et al. Pulse oximetry and peak flow as indicators of wheezing severity in children and improvement following bronchodilator treatments. Am J Emerg Med 1992;10:519-24.

25. Yamamoto LG, Wiebe RA, Rosen LM, Ringwood JW, Uechi $\mathrm{CM}$, Miller NC, et al. Oxygen saturation changes during the pediatric emergency department treatment of wheezing. Am J Emerg Med 1992;10:274-84.

26. Loren ML, Chai H, Leung P. Corticosteroids in the treatment of acute exacerbations of asthma. Ann Allergy 1980;45:67-71.
27. Rowe BH, Keller JL, Oxman AD. Effectiveness of steroid therapy in acute exacerbations of asthma: a meta-analysis. Am J Emerg Med 1992;10:302-10.

28. Waing WW. The history and physical examination. In: Cherniak $\mathrm{K}$, ed. Disorders of the respiratory tract in children. Philadelphia: WB Saunders; 1983. p. 56-77.

Endereço para correspondência:

Dra. Maria Luisa Zocal Paro

Rua Bonsucesso, 138 - São José do Rio Preto - SP

CEP 15080-430

E-mail:maluparo@uol.com.br

Fone: (17) 227.6208 - Fax: (17) 229.1197 